\title{
Electroencephalographical investigation of drinking green tea to the human brain
}

\author{
Anjaly Srimanothip ${ }^{1}$, Phakkharawat Sittiprapaporn² \\ ${ }^{1}$ Researcher, Brain Science and Engineering Innovation Research Group, School of Anti-Aging and Regenerative \\ Medicine, Mae Fah Luang University, Bangkok, Thailand, and Department of Anti-Aging Medicine, School of Anti-Aging \\ and Regenerative Medicine, Mae Fah Luang University, Bangkok, Thailand, ${ }^{2}$ Assistant Professor and Head, Brain \\ Science and Engineering Innovation Research Group, School of Anti-Aging and Regenerative Medicine, Mae Fah \\ Luang University, Bangkok, Thailand, and Department of Anti-Aging Science, School of Anti-Aging and Regenerative \\ Medicine, Mae Fah Luang University, Bangkok, Thailand
}

\section{A B S T R A C T}

Background: Nowadays green tea has become one of the most popular drinks all over the world. Evidences suggest that green tea become second most consumer following regular drinking water. Green tea contains Theanine, an amino acid primarily found in green tea and black tea. The content is generally found $1-2 \%$ of dried tea or around 60 milligrams per 200 milliliters of tea drink. In particular, this substance is found in the form of L-theanine that can be absorbed to the blood system and then to the brain within 30 minutes, which causes relaxation and reducing stress. Aims and Objective: The objective was to examine the effect of green tea consumption on electroencephalographic (EEG) activities. Materials and Methods: This study was an experimental design. The participants were twelve Thai healthy, aged between 21-55 years old. During the experiment, all participants were asked to drink green tea every 10 minutes. Results: The influence to brainwave and attentive process were tested by experimental design. The study indicated that alpha brainwave was shown after drinking green tea. Frequency of alpha brainwave along cumulative drinking of green tea was shown significantly since the $10^{\text {th }}$ minute. However, spectrum of the alpha brainwave changed significantly after drinking green tea, after $30^{\text {th }}$ minutes. Conclusion: The benefit of this study was to provide the guideline for planning and campaigning green tea consumption for the purpose of stress reduction resulting in relaxation.

Access this article online Website:

http://nepjol.info/index.php/AJMS DOI: 10.3126/ajms.v11i1.26530 E-ISSN: 2091-0576 P-ISSN: $2467-9100$

Key words: Green Tea; Brain; Brainwaves; Relaxation

\section{INTRODUCTION}

The brain is the largest electricity generator in the body. Brain electricity can be defined as brainwaves. The variety of brainwaves is influenced by neurotransmitters-neurochemical that affects neuron function-that could affect health promotion in various aspects. Four types of brainwaves have to be recognized, Beta, Alpha, Theta and delta. ${ }^{1}$ Each type of brainwave could be affected by nutrients or substances from foods or beverages. ${ }^{2}$ This effect could promote regeneration or degeneration of total health benefits.

Tea has broad health benefits from green tea to black tea due to its plant chemicals. Catechin is the main compound which is found in green tea whereas Theaflavin is mainly found in black tea. Not only theaflavin is found in black tea but thearubigins, Theobromine, Theophylline, Theanine and caffeine also. ${ }^{3,4} \mathrm{Tea}$, Camilliasinensis, is one of the most popular beverages consumed in the world. There are many types of tea which differentiate by fermentation process. Approximately three billion kilogrammes of tea is produced and consumed yearly. Many kinds of tea are consumed in different parts of the world. Green tea, which is $20 \%$ of consumption, is favored in Japan and China. $78 \%$ of tea consumption is black tea, which is consumed in Western countries and $2 \%$ is Oolong tea which is produced (partially fermented) mainly in southern China. ${ }^{3}$ Nowadays green tea has become one of the most popular drinks all over the world. Evidences suggest 
that green tea become second most consumer following regular drinking water. Green tea contains Theanine, an amino acid primarily found in green tea and black tea. The content is generally found $1-2 \%$ of dried tea or around 60 milligrams per 200 milliliters of tea drink. In particular, this substance is found in the form of L-theanine that can be absorbed to the blood system and then to the brain within 30 minutes, which L-theanine that relaxation and reducing stress. These kinds of components make various benefits for health such as antioxidant, anticancer, antiatherosclerosis effect, moreover, brain health promotion such as Alzheimer's disease, Parkinson even increase attention., ${ }^{5,6} \mathrm{~A}$ review by Ruxton summarized 23 studies on the impact of caffeine on cognitive function finding positive effects on mood, alertness, mental performance at acute intake $37.5-450 \mathrm{mg}{ }^{6}$

Electroencephalography (EEG) is traditionally used to measure the brainwaves. Each type of brainwave is associated with one's state of consciousness and different mood state. From all types of brainwave, beta brainwave was seen in normal activities as well as stressful conditions or difficulties in mental concentration. ${ }^{7-10}$ On the other hand, alpha brainwave is seen in wakefulness, relaxed, effortless and alertness condition. Therefore, persons with high alpha brainwave and low in beta brainwave could indicate status of relaxation, arousal, less stress and better concentration. ${ }^{11-15}$ In the present study, the measurement of brainwaves of accumulative drinking of green tea were done by the effectiveness of commercially available electroencephalographic (EEG) device, eego ${ }^{\mathrm{TM}}$ mylab. The benefit of this study was to provide the guideline for planning and campaigning green tea consumption for the purpose of stress reduction resulting in relaxation.

\section{MATERIALS AND METHODS}

\section{Participants}

The experiment was conducted with twelve volunteers aged between 21-55 years. All healthy participants were with no underlying disease, no history of brain surgery, no congenital illness, and not taking medicines or drugs that affect to nervous system. The protocol of this study was approved by the Ethical Committee of Mae Fah Luang University, Thailand.

\section{Tools and equipment}

Recording personal information, namely, age, gender, nationality and history of illness were recorded. The electroencephalography (EEG) was applied in this study. The neuroheadset was used to display the output, analyze and record EEG power spectrum. In this study, the effectiveness of commercially available electroencephalographic (EEG) device, eego ${ }^{\mathrm{TM}}$ mylab (ANT Neuro, Hengelo, Natherlands), was applied. The eego ${ }^{\mathrm{TM}}$ mylab is ideal for investigating all kinds of EEG paradigms. The eego ${ }^{\mathrm{TM}}$ mylab comes in four variants for recordings from 32 to 256 EEG channels. The number of channels can be easily increased by adding additional 64-channel amplifiers to the setup. Additionally, each of the 4 variants can be easily extended for simultaneous recordings with of up to 24 EMG channels or to a combination of EMG channels with a variety of physiological sensors from both the brain and body (Figure 1).

\section{Statistical analysis}

Analyzed the basic demographic data of participants by using descriptive analysis with qualitative data summarize in term of frequency and percentage, with quantitative data summarized in term of average and standard deviation. Use inferential statistic compared each type of EEG power spectra of before and after experiment by compared $t$-test while performing the accumulative drinking of green tea. Every test was set to the statistic significant at $p<0.05$.

\section{RESULTS}

Based on Table 1 Figure 2, it was found that delta and theta brainwaves gradually declined while alpha, beta and gamma

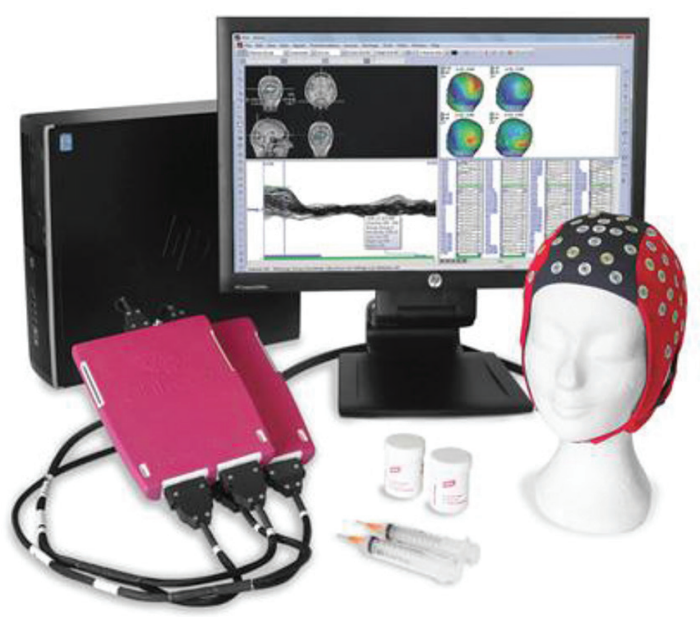

Figure 1: Electroencephalographic device, eego ${ }^{\mathrm{TM}}$ mylab (ANT Neuro, Hengelo, Natherlands)

\begin{tabular}{|c|c|c|c|c|c|}
\hline \multirow[t]{2}{*}{ EEG activities } & \multicolumn{2}{|c|}{ Before } & \multicolumn{2}{|c|}{ After } & \multirow[t]{2}{*}{$p$-value } \\
\hline & Mean & SD & Mean & SD & \\
\hline Delta brainwave & 0.46 & 0.09 & 0.12 & 0.78 & 0.35 \\
\hline Theta brainwave & 0.59 & 0.37 & 0.16 & 0.65 & 0.38 \\
\hline Alpha brainwave & 1.91 & 0.19 & 2.53 & 0.15 & $0.01^{*}$ \\
\hline Beta brainwave & 0.11 & 0.87 & 0.34 & 0.27 & 0.91 \\
\hline Gamma brainwave & 0.34 & 0.18 & 0.52 & 0.67 & 0.14 \\
\hline
\end{tabular}




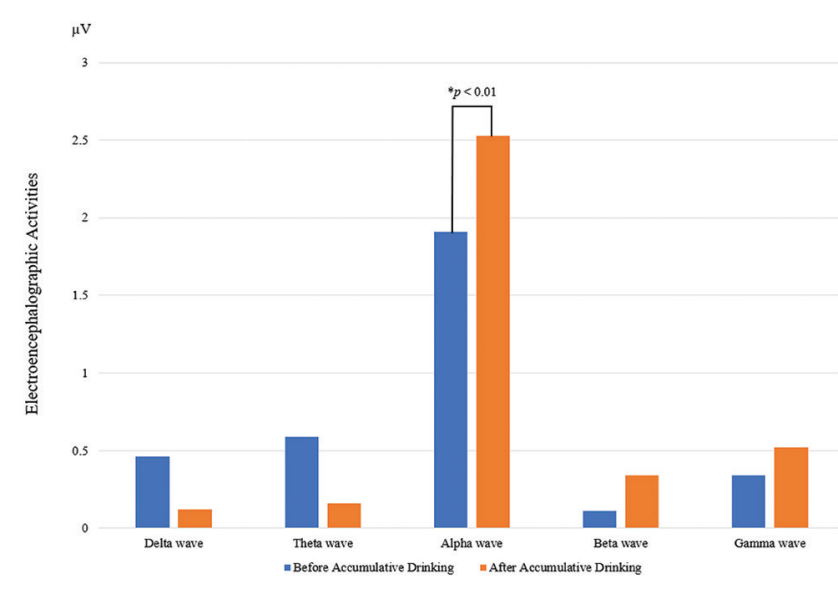

Figure 2: Comparison of electroencephalographic activities comparing between before and after accumulative drinking of green tea

brainwaves increased. Only alpha brainwave increased after accumulative drinking of green tea with statistically significant at the 0.05 level. In all of these brainwaves, alpha brainwave increased highly after accumulative drinking of green tea compared to other frequency bands.

More specifically, delta brainwave decreased after accumulative drinking of green tea with no statistically significant (before drinking: 0.46 $\pm 0.09 \mu \mathrm{V}$; after drinking: $0.12 \pm 0.78 \mu \mathrm{V} ; \mathrm{t}(11)=1.19 ; p=0.35)$. Similar to delta brainwave, theta brainwave decreased after accumulative drinking of green tea with no statistical significant (before drinking: $0.59 \pm 0.37 \mu \mathrm{V}$; after drinking: $0.16 \pm 0.65 \mu \mathrm{V}$; $\mathrm{t}(11)=0.90 ; p=0.38)$. On the other hand, it was found that alpha brainwave was found to be increased with statistically significant at the 0.05 level after accumulative drinking of green tea (before drinking: $1.91 \pm 0.19 \mu \mathrm{V}$; after drinking: $2.53 \pm 0.15 \mu \mathrm{V} ; \mathrm{t}(11)=0.17 ; p=0.01)$.

However, it was found that beta brainwave was not changed after accumulative drinking of green tea (before drinking: $0.11 \pm 1.87 \mu \mathrm{V}$; after drinking: $0.34 \pm 0.27 \mu \mathrm{V}$; $\mathrm{t}(11)=1.37 ; p=0.91)$ the same as gamma brainwave which was also not changed after accumulative drinking of green tea (before drinking: $0.34 \pm 0.18 \mu \mathrm{V}$; after drinking: $0.52 \pm 0.67 \mu \mathrm{V} ; \mathrm{t}(11)=0.73 ; p=0.14)$.

\section{DISCUSSION}

The aim of the current study was to find out whether there was an influence of green tea on brainwave and attentive process in a accumulative drinking period. In the current study, the influence to brainwave and attentive process were tested by experimental design. The study indicated alpha brainwave was shown after drinking green tea. Interestingly, frequency of alpha brainwave along accumulative drinking of green tea was shown significantly since the $10^{\text {th }}$ minute; however, spectrum of the alpha brainwave changed significantly after drinking green tea, after $30^{\text {th }}$ minutes. The influence of green tea in this study to attention associated with a study of Bruin that black tea improved reaction times on the sensory-attention test, ${ }^{16}$ nevertheless, not associated with Bruin's study in accuracy. Accordingly, alpha brainwave was still associated with alertness and peacefulness ${ }^{17,18}$ and corresponded to memory storage and processing. ${ }^{1}$ Alpha brainwave in current study was dominantly shown on $\mathrm{Fz}$ position where associated with working memory and also attention, according to Broadmann cortical area. ${ }^{19}$

This effect of alpha brainwave could be associated with attention on doing spatial test together ingredients from Theanin, ${ }^{16}$ which is known to help attention and increase alpha wave activity according to a study of Gomez-Ramirez and Owen. ${ }^{20}$ Alpha brainwave has shown on Fz position, though, it is also shown on $\mathrm{Cz}$ position which corresponded to somato association cortex, according to Broadmann cortical area. ${ }^{19}$ This position related to working memory from visual and visuomotor attention. ${ }^{19}$ Spectrums of alpha brainwave from this position were associated with doing visual spatial test of participants.

Interestingly, caffeine is known to inhibit neurotransmitters that slow down brain activity and influence others that alter mental performance such as noradrenaline, dopamine and serotonin as the study from Fredhlom. ${ }^{6}$ All this information was the reason for this study to study brainwaves and attentive processes in accumulative drinking of green tea in order to promote longevity and health. This study had been set to prove the hypothesis that brainwaves could be changed after cumulative drinking green tea.

\section{CONCLUSION}

It was found that the means of the alpha brainwave of the samples were increased. The accumulative drinking of green tea in this study might affected to the samples nervous systems including the central and automatic nervous systems. This way of accumulative drinking influenced the central nervous system by making the samples significantly felt happy and relaxed. The accumulative drinking of green tea could help promote alpha brainwave which help relaxing and attention. This could promote longevity for brain health in the term of improving attention with no stress.

\section{ACKNOWLEDGEMENT}

This research was supported by research funding from Mae Fah Luang University grant (Electroencephalogram 
Laboratory 2019), Brain Science and Engineering Innovation Research Group, Mae Fah Luang University grant (2019) (MFU-grant no. 611U109005) and 2020, Thailand. We thank all of the subjects involved in this research as main data provider of this studies.

\section{REFERENCES}

1 Braverman E, Watt TJ and Bajaj A. Cognitive diseases \& impairments: diagnostic, prevention and therapeutic interventions. Health Practitioner's Guide to Anti-aging \& Regenerative Medicine 2012, pp. 209-262.

2. de Jager CA and Kovatcheva A. Summary and Discussion: Methodologies to assess long term effects of nutrition on brain function. Nutrition Review 2010; 68(suppl. 1): S53-S58.

3. Khan $\mathrm{N}$ and Mukhtar $\mathrm{H}$. Tea polyphenols for health promotion. Life science 2007; 81: 519-533.

4. Pinto MS. Tea: A new perspective on health benefits. Food research International 2013; 53(2): 558 - 567

5. Sharangi AB. Medicinal and therapeutic potentialities of tea (Camilliasinensis L.): A review. Food Research International 2009; 42: 529-535.

6. Ruxton CHS. The health effect of black tea and flavonoids. Nutrition and Food Science 2009; 39(3): 283-294.

7. Kraaier V, Van Huffelen AC and Wieneke GH. Changes in quantitative EEG and blood flow velocity due to standardized hyperventilation; a model of transient ischaemia in young human subjects. Electroencephal Clin Neurophysiol 1988; 70: 377-387.

8. Kraaier V, Van Huffelen AC, Wieneke GH, Van der Worp HB and Bär PR. Quantitative EEG changes due to cerebral vasoconstriction. Indomethacin versus hyperventilationinduced reduction in cerebral blood flow in normal subjects. Electroencephal Clin Neurophysiol 1992; 82: 208-212.
9. Kubitz KA and Mott AA. EEG power spectral densities during and after cycle ergometer exercise. Res Quart Exercise and Sport 1996; 67(1): 91-96.

10. Kubitz KA and Pothakos K. Does aerobic exercise decrease brain activation? J Sport and Exercise Psychol1997; 19: 291- 301.

11. Nielsen B, Hgldig $T$, Bidstrup $F$, Gonzalez-Alonso $J$ and Christoffersen GRJ. Brain activity and fatigue during prolonged exercise in the heat. Eur J Appl Physiol 2001; 442: 41-48.

12. Nybo $L$ and Nielsen B. Perceived exertion is associated with an altered brain activity during exercise with progressive hyperthermia. J Appl Physiol 2001; 91: 2017-2023.

13. Wasee P. Family Doctor. Bangkok: Komolkeemthong Foundation Press, 1978.

14. Wongwattana P. Massages for Curing Diseases. Bangkok: Holistic Publishing, 1996.

15. Samcharoen P. Massages for Health: National Institute of Thai Traditional Medicine. Bangkok: Department of Medical Services, Ministry of Public Health, 2009.

16. De Bruin EA, Rowson MJ, Van Buren L, Rycroft JA and Owen GN. Black tea improves attention and self-reported alertness. Appetite 2011; 56(2): 235-340.

17. Sittiprapaporn W. Neurofeedback: Use technology to shape the mind. In short term program, July $13-14,2013$, School of Anti-Aging and Regenerative Medicine, Mae Fah Luang University, Bangkok, Thailand, 2013.

18. Huang $T L$ and Charyton C. A comprehensive review of physiological effects of brainwave entrainment. Alternative Therarpies Health 2008; 14(5): 38-50.

19. Cortical Functions, Trans Cranial Technologies Idt. (n.d.). [Online] 2015 [Cited 2014 April 8. Available from http://www.transcranial. com/local/manuals/cortical_functions_ref_v1_0_pdf.pdf

20. Bryan J, Tuckey M, Einöther SJ, Garczarek U, Garrick A and De Bruin EA. Relationships between tea and other beverage consumption to work performance and mood. Appetite 2012; 58(1): 339-346.

\section{Authors Contribution:}

AS- Concept and design of the study, statistically analyzed and interpreted; PS- Concept and design of the study, statistically analyzed and interpreted,

manuscript preparation, critical revision of the manuscript.

\section{Work attributed to:}

Brain Science and Engineering Innovation Research Group

School of Anti-Aging and Regenerative Medicine, Mae Fah Luang University, Thailand

\section{Orcid ID:}

Dr. AnjalySrimanothip - (D) https://orcid.org/0000-0003-0455-4281

Dr. Phakkharawat Sittiprapaporn - (1) https://orcid.org/0000-0002-4103-9396

Source of Support: Mae Fah Luang University grant (Electroencephalogram Laboratory 2019), Thailand; Brain Science and Engineering Innovation Research Group, Mae Fah Luang University grant (2019) (MFU-grant no. 611U109005) and 2020, Thailand. Conflict of Interest: None. 